\title{
CaseGuide: Making Cheap Smartphones Accessible to Individuals with Visual Impairments in Informal Settlements
}

\author{
ROOS VAN GREEVENBROEK, UCL Interaction Centre, University College of London
}

\begin{abstract}
Individuals with visual impairments in informal settlements (IVIIS) depend highly on others for access to basic services. Smartphones can help provide assistive technology and access to basic services but are too expensive for IVIIS or lack accessibility features. This study explores and promotes a low-cost concept that uses a static interface overlay app in conjunction with a button enabled phone case, to enable the use of cheap smartphones and increase IVIIS autonomy and inclusion in society. Using existing research and an observational study of YouTube videos, design requirements were determined. A low-fidelity prototype was developed and usertested on one visually impaired and two blindfolded participants. Although usertests showed promising results, research and usertesting were limited. Future research and usertests with IVIIS are needed to validate if CaseGuide is a desirable solution for IVIIS.
\end{abstract}

CCS Concepts: • Human-centered computing $\rightarrow$ Accessibility technologies.

Additional Key Words and Phrases: individuals with visual impairments, smartphones, assistive technology, informal settlements

ACM Reference Format:

Roos van Greevenbroek. 2020. CaseGuide: Making Cheap Smartphones Accessible to Individuals with Visual Impairments in Informal Settlements. 1, 1 (July 2020), 6 pages.

The majority of individuals with visual impairments (IVI's) live in low-income areas [23] such as informal settlements (unauthorised construction of housing), where less than 5-15\% can access assistive technologies (AT's) due to poverty and unregulated basic services $[2,11,16]$. IVI's in informal settlements (IVIIS) depend on others for navigation, employment, education, finances and social contacts [3-5,23] which can impose exclusion and poverty [2, 4]. Smartphones can play a life-changing role in providing AT to IVI's and accessing education, social contacts and employment, supporting well-being and inclusion in society $[2,3,12]$. Although mobile phone penetration rates and internet coverage are high, smartphones are not yet common in informal settlements $[2,24]$. Affordable smartphones (under 50 dollars) lack basic accessibility features, such as screenreaders IVI's depend on during use, or have prohibiting usability failures, limiting their usefulness $[2,4,9,11,19]$. The inaccessibility of affordable smartphones, steep learning curve, digital illiteracy and low income [19, 20,23] cause IVIIS to use affordable basic/feature phones with tangible keypads so they can execute simple actions themselves [2,3]. However, basic phones cannot provide AT, most interactions require help from others (putting IVI's in a vulnerable position), and smartphone use in low-income countries is expected to triple in the next 5 years while basic phones availability will decline [2, 3, 20], driving the need to adapt. Multiple low-cost solutions adding accessibility features to mobile phones have been explored such as Seeing AI, but are incompatible with cheaper models, too costly, and do not guide operating smartphones in general, which likely becomes the biggest challenge for IVIIS.

Author's address: Roos van Greevenbroek, roos.greevenbroek.19@ucl.ac.uk, UCL Interaction Centre, University College of London, 66-72 Gower Street, London, UK, WC1E 6BT.

Permission to make digital or hard copies of all or part of this work for personal or classroom use is granted without fee provided that copies are not made or distributed for profit or commercial advantage and that copies bear this notice and the full citation on the first page. Copyrights for components of this work owned by others than ACM must be honored. Abstracting with credit is permitted. To copy otherwise, or republish, to post on servers or to redistribute to lists, requires prior specific permission and/or a fee. Request permissions from permissions@acm.org.

(c) 2020 Association for Computing Machinery.

Manuscript submitted to ACM

Manuscript submitted to ACM 
Therefore, this study explores how a low-cost solution can enhance accessible use of cheap smartphones to provide IVIIS with affordable access to AT and promotes a concept that uses a static interface overlay app in conjunction with a buttoned phone case. This solution could ease smartphone adoption and increase autonomy and social participation.

\section{LITERATURE REVIEW}

Earlier research on smartphone accessibility features for IVI's was leveraged to identify difficulties and design suggestions. Most importantly, the absence of tactile feedback challenges correct positioning on screens and interactions [8, 20]. Simple and important buttons, with different sizes and shapes, fixed locations and marks to navigate from, should be provided [7, 17]. Secondarily, smartphones often lack clear (audio) feedback [8, 20], where screen readers, reading everything out loud, limit privacy $[8,20]$. Research suggests that vibration and sonification can give additional feedback on distinct situations [7] and separate buttons should be provided to mute screen readers [17] and activate voice command mode [17]. Thirdly, gestures and accessibility features function inconsistent across apps and phones [8, 20], making it unclear how to interact. Previous research advises to hierarchically structure menus and actions, and make gestures universal $[7,17]$. The most promising research on increasing smartphone accessibility affordably concerns developing personalisable static interface overlays [15, 21]. The overlay structures every screen and app into the same simple, consistent, personalised UI. Although IVI's found this a useful solution, physical affordances such as buttons are still absent; a much-expressed need of IVI's [8, 17, 21]. This sparked the interest to explore the concept of combining a static interface overlay while providing tactile buttons on cheap smartphones using a phone case.

\section{RESEARCH AND REQUIREMENTS}

To complement findings from earlier research with contextual information, an observational study analysing YouTube video content was conducted. This method seemed promising as it was successfully used in research on smartphone accessibility with people with disabilities[1] and IVIIS can access YouTube through NGO led projects [13]. Terms (phone, visual impairment and informal settlements) and their synonyms were combined to select and review 138 videos (2010 to 2020) featuring IVIIS' environment and phone use, of which 34 relevant videos were analysed (252 minutes) using Braun and Clark's six phased inductive thematic analysis [6] where four themes emerged. To ensure valid interpretation of the observations, 12 videos were independently peer-reviewed where differences in codes and themes were discussed.

Phones were perceived as 'success objects'. In only a few videos IVIIS used mobile phones, possibly exemplifying the lack of access. The videos mostly featured basic phones and focused on the accomplishment of IVI's independent use of the phone. Similar to earlier research [7, 17], differently shaped buttons and basic keypads enabled basic interactions.

IVI's suffered exclusion due to 'AT costs and compatibility'. Although primary schools educate braille, IVIIS were excluded from higher education as AT was missing owing to high costs and need for installation on compatible phones.

Similar to research [4], the 'environment of informal settlements' with large height differences and the littered ground was difficult for IVIIS and unsuitable for fragile touchscreens. Many residents used protective phone cases.

IVIIS often held a 'vulnerable position'. Some IVIIS expressed anxiety about being exploited by others because of their vulnerability. Almost no VIP used white canes as they were too expensive and sometimes stolen from them.

Based on the results of earlier research and the YouTube video study user requirements were formed. The concept should support: (1) interaction by providing different tactile affordances with fixed locations, (2) easy locating of important buttons (e.g. screen reader, voice command), (3) important functions (managing finances) by providing a consistent UI, menu and gestures, (4) simple and affordable software and hardware, compatible with cheaper smartphones, (5) protection of smartphones from theft and the environment.

Manuscript submitted to ACM 


\section{DESIGN'CASEGUIDE'}

First, flows were made of important tasks that IVIIS could not perform independently [4] to identify needed commands and structures. Exploring sketches were made and reviewed based on user requirements, energy consumption, affordability and support of important tasks and led to the design of 'CaseGuide'.

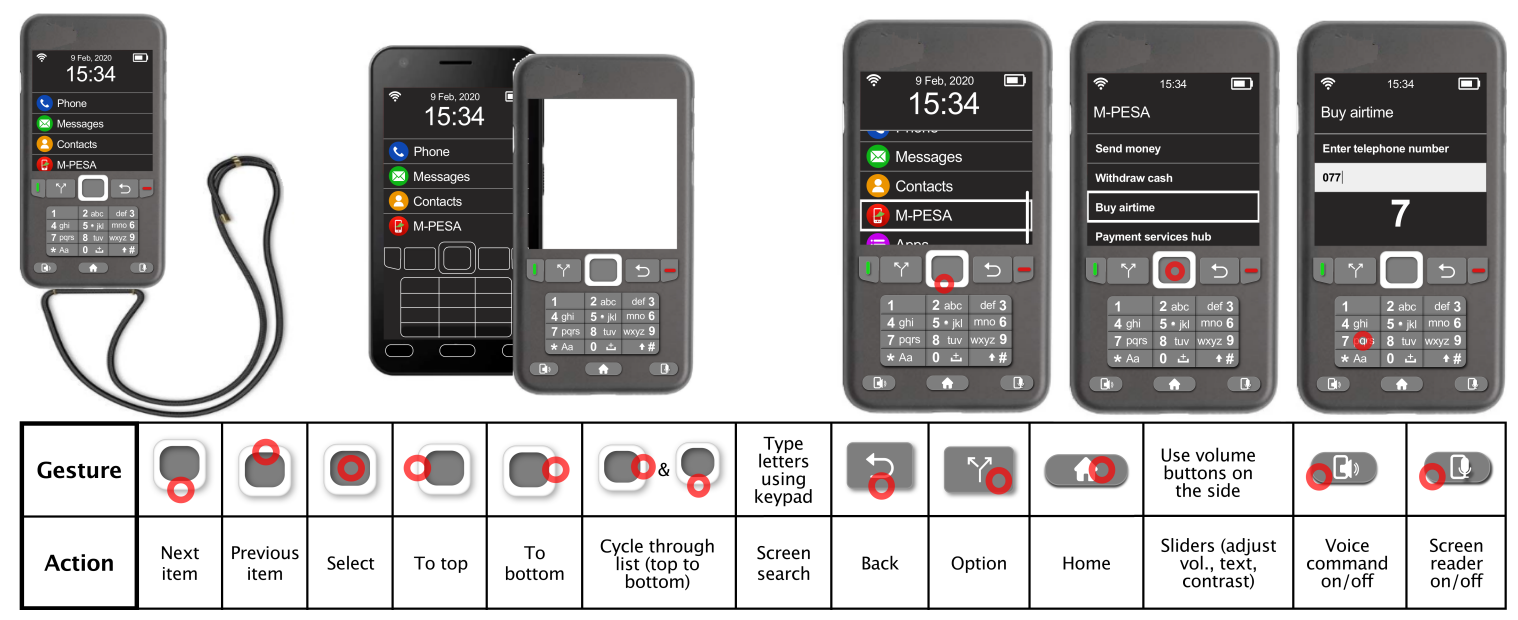

Fig. 1. Design and interactions of the CaseGuide concept

CaseGuide, designed for the most purchased cheap smartphone in Kenya (Neon Kicka 4 [10]), consists of a launcher app and a silicone phone case with a basic keypad, that preserves a part of the smartphones' touchscreen to allow partially sighted people use without a screen reader. The app reformats screens hierarchically and visually accessibly for IVI's and saves fixed positions on the screen to calibrate with the keypad of the phone case, allowing control over the smartphone through the buttoned case. The basic keypad, known to IVIIS, help transition from basic phones to smartphones. Differently shaped and raised buttons, with raised dots on number five, help to distinguish and locate keys while typing. IVI's can navigate using the four-dimensional button, back and home button and simple commands (see Figure 1). Extra buttons for screen reader and voice command allow easy localisation and (de)activation. An 'option' button was added to always make next the actions available, preventing IVI's from being blocked. The key-cord on the phone case can affordably prevent smartphones from theft. The digital UI, large white text on black background, grants high contrast and consumes less energy, and can be personalised in the settings. The home screen displays time, battery level, and most-used apps. Information on screens is vertically listed, simplifying up and down navigation using the four-directional button and limiting cognitive load. When selecting an option, one list of next possible actions at the time is presented, which is repeated until the desired goal is reached. This hierarchical structure applies to all apps and can help IVI's navigate. To give additional feedback when typing, each number or letter enlarges briefly (see Figure 1).

\section{PROTOTYPE TESTING AND RESULTS}

To gain insight on users' difficulties and needs on the phone cases' design, static overlay UI, and interactions, a Wizard-of-Oz usertest was conducted with one European participant with severe near-sightedness and astigmatism and two sighted European participants (blindfolded to imitate blindness). Similar to IVIIS, they had no experience with assistive features and used basic keypads before. A clickable digital prototype of the launcher app was created 
and presented on a smartphone wrapped in a paper prototype, mocking the phone case and button interactions while the researcher mimicked screen reader feedback. Participants executed an unfamiliar task to rule out interference of previous knowledge: buying phone credits using M-PESA, an important activity for IVIIS [4]. During the session, structured notes were made to capture observations and relevant participants' feedback verbatim.

Participants were positive on the design of the phone case (P2 "It is nice to feel what I'm pressing and to control the smartphone this way"). The basic keypad with raised keys and dots helped to locate, distinguish and navigate between buttons. Positions of new buttons came naturally, although participants needed longer to understand their functions. Separate screen reader and voice command buttons were found very useful, particularly to mute the screen reader when reading an M-PESA text out loud resonating with suggestions from previous studies [7, 8, 17, 20]. The UI of the static overlay was perceived as helpful and participants could independently perform their task. Similar to earlier research $[7,15,17,21]$, participants benefited from the hierarchy and continuity of the static overlay, and the low amount of information per screen. It helped clarify expectations, actions and reduced cognitive load (P3 "The screenreader is so short I quickly know where I am"). Participants doubted whether it could be implemented in complex apps. The near-sighted participant experienced reading difficulties (P1 "I need bigger text and way more contrast, blue and white would be better for $m e^{\prime \prime}$ ), exemplifying the need for UI personalisation. Using consistent and universal gestures, as earlier studies suggested $[8,20]$, helped participants interact as most were similar to their past experiences and easily recollected. Going to the top (press left) or bottom (press right) was intuitive (P1 "fust like on the remote control") but scrolling through lists (press right and down) was new and more difficult. Participants also experienced difficulties in distinguishing different functions of the buttons controlling volume, text size and contrast. They also wanted more haptic/sound feedback on received notifications, battery levels and navigation to be less dependent on the screen reader.

\section{DISCUSSION}

This research explored a novel low-cost solution to enable affordable access to cheap smartphones and AT through 'CaseGuide'; a launcher app that reformats screens hierarchically and visually accessibly for IVI's and works in conjunction with a buttoned phone, enhancing smartphone control. CaseGuide could help IVIIS adopt and use cheap smartphones easily and independently. The static overlay can also increase existing apps' accessibility such as M-PESA, which was first unusable for IVI's [4]). With CaseGuide, IVI's can also access apps that offer assistive technology, like Seeing AI [14], which can unlock more possibilities for IVIIS on access to navigation, information and education.

Usertests showed positive results on easy control of a smartphone through CaseGuide, opportunities to improve on interaction and feedback CaseGuide, and uncovered the need to test the implementation of the static overlay on complex apps on feasibility and accuracy. However, because of COVID-19, usertesting was restricted to a few, not quite representative participants, characterising findings and ignoring contextual and social information about IVIIS [18]. Conducting interviews and surveys with IVIIS was impossible. Research, therefore, was limited and subpar as it relied on the analysis of literature and YouTube video content mostly uploaded by local news networks, which possibly distorted the representation of IVIIS' behaviours and needs. The choice to focus on affordability was at the expense of the flexibility and efficiency of the concept. The phone case might only fit the Neon Kicka 4 smartphone or similar. Although the app is designed to save energy, smartphone batteries need frequent recharging, which is limited in informal settlements [25]. Therefore, smartphones may not run continuously and IVIIS cannot fully rely on smartphone-enabled AT. Limitations in research and participants demand future research with IVIIS to investigate whether CaseGuide actually provides a desirable solution for IVIIS. Social infrastructures need to be created to support and embed the development of this solution in this community to make its effect successful [22].

Manuscript submitted to ACM 
CaseGuide: Making Cheap Smartphones Accessible to Individuals with Visual Impairments in Informal Settlements 5

\section{REFERENCES}

[1] Lisa Anthony, YooJin Kim, and Leah Findlater. 2013. Analyzing User-Generated Youtube Videos to Understand Touchscreen Use by People with Motor Impairments. In Proceedings of the SIGCHI Conference on Human Factors in Computing Systems (CHI '13). Association for Computing Machinery, New York, NY, USA, 1223-1232. https://doi.org/10.1145/2470654.2466158

[2] Clara Aranda-Jan and Alizee Boutard. 2019. Understanding the Mobile Disability Gap. https://www.gsma.com/mobilefordevelopment/wpcontent/uploads/2019/12/GSMA_Understanding-the-mobile-disability-gap_116pg_Accessible.pdf

[3] Katy Arnold. 2019. The Social Network: Mobile Phone Use of Visually Impaired People in Kibera, Kenya. Master's thesis.

[4] Giulia Barbareschi, Catherine Holloway, Katherine Arnold, Grace Magomere, Wycliffe Ambeyi Wetende, Gabriel Ngare, and Joyce Olenja. 2020. The Social Network: How People with Visual Impairment Use Mobile Phones in Kibera, Kenya. In Proceedings of the 2020 CHI Conference on Human Factors in Computing Systems (CHI '20). Association for Computing Machinery, New York, NY, USA, 1-15. https://doi.org/10.1145/3313831.3376658

[5] Julia Bird, Piero Montebruno, and Tanner Regan. 2017. Life in a slum: understanding living conditions in Nairobi's slums across time and space. Oxford Review of Economic Policy 33, 3 (2017), 496-520. https://doi.org/10.1093/oxrep/grx036

[6] Virginia Braun and Victoria Clarke. 2006. Using thematic analysis in psychology. Qualitative research in psychology 3, 2 (2006), 77-101. https: //doi.org/10.1191/1478088706qp063oa

[7] Sarah Chiti and Barbara Leporini. 2012. Accessibility of Android-Based Mobile Devices: A Prototype to Investigate Interaction with Blind Users. In Proceedings of the 13th International Conference on Computers Helping People with Special Needs - Volume Part II (ICCHP'12). Springer-Verlag, Berlin, Heidelberg, 607-614. https://doi.org/10.1007/978-3-642-31534-3_89

[8] Rafael Jeferson Pezzuto Damaceno, Juliana Cristina Braga, and Jesús Pascual Mena Chalco. 2016. Mobile Device Accessibility for the Visually Impaired: Problems Mapping and Empirical Study of Touch Screen Gestures. In Proceedings of the 15th Brazilian Symposium on Human Factors in Computing Systems. Association for Computing Machinery, New York, NY, USA, Article Article 2, 10 pages. https://doi.org/10.1145/3033701.3033703

[9] Joao de Sousa e Silva, Antonio Pereira, Ramiro Goncalves, and Silvia Gomes. 2014. State of the art of accessible development for smart devices: From a disable and not impaired point of view. In 2014 9th Iberian Conference on Information Systems and Technologies (CISTI). IEEE, 1-5. https: //doi.org/10.1109/CISTI.2014.6876937

[10] GSMA. 2017. Accelerating Affordable Smartphone Ownership in Emerging Markets. https://www.gsma.com/mobilefordevelopment/resources/ accelerating-affordable-smartphone- ownership-in-emerging-markets/

[11] GSMA. 2018. Leveraging the Potential of Mobile for Persons with Disabilities. https://www.gsma.com/mobilefordevelopment/resources/leveragingthe-potential-of-mobile-for-persons-with-disabilities/

[12] Hakobyan, Jo Lumsden, Dympna O’Sullivan, and Hannah Bartlett. 2013. Mobile assistive technologies for the visually impaired. Survey of ophthalmology 58, 6 (2013), 513-528. https://doi.org/10.1016/j.survophthal.2012.10.004

[13] Christopher Huggins and Natasha Frosina. 2017. ICT-driven projects for land governance in Kenya: Disruption and e-government frameworks. Geofournal 82, 4 (2017), 643-663. https://doi.org/10.1007/s10708-016-9710-6

[14] Steven Kelley. 2018. Seeing AI: Artificial Intelligence for Blind and Visually Impaired Users. https://visionaware.org/everyday-living/helpfulproducts/using-apps/seeing-ai-app/

[15] Akif Khan, Shah Khusro, and Iftikhar Alam. [n.d.]. Blindsense: An accessibility-inclusive universal user interface for blind people. Engineering, Technology \& Applied Science Research 8, 2 ([n.d.]), 2775-2784. https://www.etasr.com/index.php/ETASR/article/view/1895

[16] Rebecca Matter, Mark Harniss, Tone Øderud, Johan Borg, and Arne H. Eide. 2016. Assistive technology in resource-limited environments: a scoping review. Disability and Rehabilitation: Assistive Technology 12 (07 2016), 1-10. https://doi.org/10.1080/17483107.2016.1188170

[17] Na Mi, Lora Cavuoto, Kenneth Benson, Tonya Smith-Jackson, and Maury Nussbaum. 2013. A heuristic checklist for an accessible smartphone interface design. Universal Access in the Information Society 13 (11 2013). https://doi.org/10.1007/s10209-013-0321-4

[18] Rebecca Monteleone. 2018. Beyond Participation: Empowering People with Disabilities in Research and Design. Technology Innovation 20 (11 2018). https://doi.org/10.21300/20.1-2.2018.133

[19] Joyojeet Pal, Anandhi Viswanathan, Priyank Chandra, Anisha Nazareth, Vaishnav Kameswaran, Hariharan Subramonyam, Aditya Johri, Mark S. Ackerman, and Sile O'Modhrain. 2017. Agency in Assistive Technology Adoption: Visual Impairment and Smartphone Use in Bangalore. In Proceedings of the 2017 CHI Conference on Human Factors in Computing Systems (CHI '17). Association for Computing Machinery, New York, NY, USA, 5929-5940. https://doi.org/10.1145/3025453.3025895

[20] André Rodrigues, Kyle Montague, Hugo Nicolau, and Tiago Guerreiro. 2015. Getting Smartphones to Talkback: Understanding the Smartphone Adoption Process of Blind Users. In Proceedings of the 17th International ACM SIGACCESS Conference on Computers Accessibility (ASSETS '15). Association for Computing Machinery, New York, NY, USA, 23-32. https://doi.org/10.1145/2700648.2809842

[21] André Rodrigues, André Santos, Kyle Montague, and Tiago Guerreiro. 2017. Improving Smartphone Accessibility with Personalizable Static Overlays. In Proceedings of the 19th International ACM SIGACCESS Conference on Computers and Accessibility (ASSETS '17). Association for Computing Machinery, New York, NY, USA, 37-41. https://doi.org/10.1145/3132525.3132558

[22] Kentaro Toyama. 2011. Technology as amplifier in international development. In Proceedings of the 2011 iConference. 75-82. https://doi.org/10.1145/ 1940761.1940772

[23] WHO. 2016. W.H. Priority Assistive Products List (APL). https://www.who.int/phi/implementation/assistive_technology/global_survey-apl/en/. 
[24] Susan Wyche and Charles Steinfield. 2016. Why don't farmers use cell phones to access market prices? Technology affordances and barriers to market information services adoption in rural Kenya. Information Technology for Development 22, 2 (2016), 320-333. https://doi.org/doi.org/10.1080/ 02681102.2015.1048184

[25] Susan P Wyche and Laura L Murphy. [n.d.]. Powering the cellphone revolution: findings from mobile phone charging trials in off-grid Kenya. In Proceedings of the SIGCHI Conference on Human Factors in Computing Systems. 1959-1968. https://doi.org/doi.org/10.1145/2470654.2466260 